\title{
Cryptogenic organizing pneumonia
}

INSERM

\section{Source}

INSERM. (1999). Orphanet: an online rare disease and orphan drug data base.

Cryptogenic organizing pneumonia. ORPHA:1302

Cryptogenic org anizing pneumonia (COP) is a form of idiopathic interstitial pneumonia characterized pathologically by organizing pneumonia (OP) that presents with nonspecific flu-like symptoms, as well as cough and dyspnea and where no etiological agent is found. 